\title{
SOFTWARE DIDÁTICO PARA ANÁLISE DE VIBRAÇÕES EM UMA VIGA EM BALANÇO SUBMETIDA À VIBRAÇÃO HARMÔNICA
}

Vladimir Topázio Barbosa - vladi.tb@ hotmail.com*

Paula Frassinetti Cavalcante - pfc@ufba.br*

Jayann I Lira Almeida - jayann.almeida@ufba.br*

Thiago Lima Carneiro - thiagocarneiro.90@gmail.com*

*Universidade Federal da Bahia, Departamento de Engenharia Mecânica

Rua Aristides Novis, 02, Federação

40210-630 - Salvador - Bahia

Resumo: $O$ comportamento de máquinas rotativas, pela sua complexidade, exige projeto e análise adequada para os diversos componentes mecânicos que compõe a máquina. Contudo, determinar a interação de todos os subsistemas da máquina, considerando o grande número de componentes e equipamentos, não é uma tarefa fácil, uma vez que a interação dos mesmos se dá através dos diversos elementos de conexão como mancais e selos mecânicos de fluxo. Além disso, o comportamento do sistema principal, constituído do rotor e estrutura de suporte (mancais) podem inserir no sistema o efeito de diversos fenômenos tais como desalinhamento e desbalanceamento, além das vibrações transversais, torcionais e axiais apresentadas pelo rotor quando em operação. Esses fenômenos podem interferir na resposta do sistema completo da máquina (rotor-estrutura de suporte + base da máquina). Porém, os mesmos podem ser investigados através de modelos matemáticos que podem ser ajustados a partir da aquisição de dados experimentais obtidos do sistema. Assim sendo, neste artigo será apresentado o desenvolvimento, através do software LabView, de uma interface gráfica para análise dos sinais oriundos de 2 bancadas de ensaios, que será utilizada como ferramenta de apoio nas aulas de vibrações para o estudo de fenômenos em máquinas rotativas. A interface permite analisar um sistema constituído de uma viga em balanço excitada por um sistema cameseguidor, que será analisado, e um segundo sistema constituído de um eixo, acionado por um motor elétrico e suportado por dois mancais.

Palavras-chave: Máquinas rotativas. LabView. Desalinhamento. Ferramentas de ensino.

\section{INTRODUÇÃO}

O estudo das vibrações mecânicas é de extrema importância para o conhecimento dos engenheiros mecânicos que atuam principalmente na área de manutenção preditiva, considerando que os fenômenos vibratórios podem influenciar a resposta da máquina. Falhas em equipamentos que operam em condições críticas podem levar a grandes prejuízos, desde o 
aumento dos gastos com manutenção ou gastos com quebra inesperada do equipamento. Por outro lado, há tempos softwares vêm sendo desenvolvidos para facilitar o entendimento do comportamento dos equipamentos dinâmicos, através da análise dos sinais de vibrações oriundos da máquina. Estes softwares tornaram-se fundamentais para o acompanhamento dos sinais da máquina com enfoque na manutenção preditiva dos equipamentos rotativos. $\mathrm{O}$ software Labview é uma linguagem de programação gráfica baseada em fluxo de dados que utiliza ícones para criar aplicações (NATIONAL INSTRUMENTS, 2003). Os programas são chamados de instrumentos virtuais, Virtual Instruments (VI's), e são constituídos principalmente do painel frontal, que é a interface com o usuário, e do diagrama de bloco, que contém o código fonte gráfico responsável pelo funcionamento dos VI’s. A linguagem gráfica do programa, apresentada através de diagramas de blocos de funções, permitem ao usuário criar subVI's e utilizá-los em outros programas. Além disso os objetos do diagrama de bloco incluem terminais, subVI's, funções, constantes, estruturas e ligações, que transferem dados entre outros objetos. Na Figura 1 é mostrado um subVI para análise e aquisição de dados.

Figura 1 - Diagrama de blocos do SubVI para aquisição de dados.

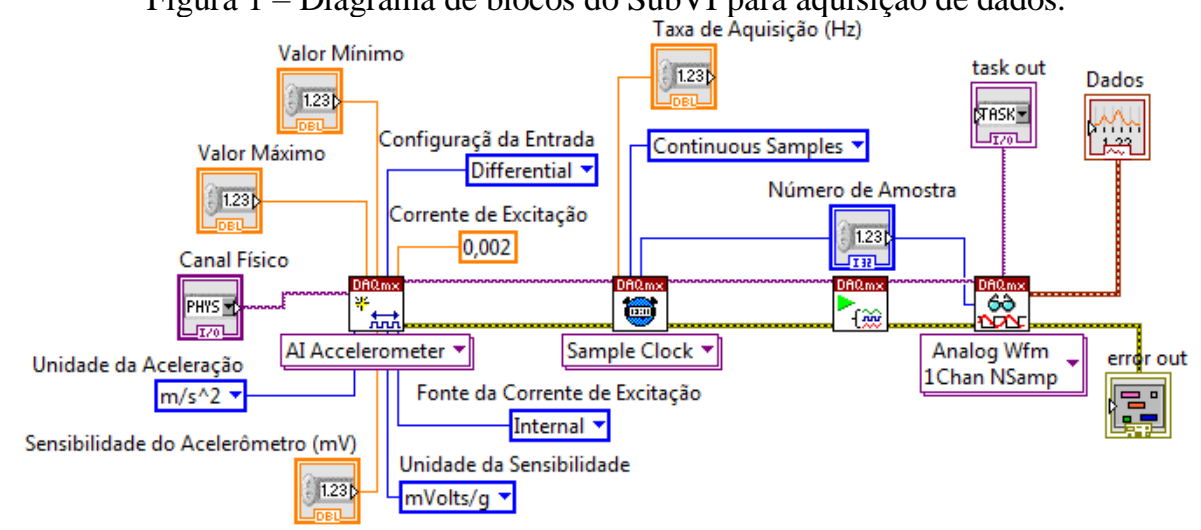

Fonte: Próprio Autor

$\mathrm{Na}$ análise do desenvolvimento do programa, o exemplo prático utilizado foi um sistema constituído de uma viga em balanço submetida a uma excitação harmônica. A extremidade livre da viga está sujeita a excitação harmônica oriunda de um sistema came-seguidor que transforma o movimento de rotação em movimento alternado (MELO; CARNEIRO, 2008). Para análise do comportamento da viga, foi considerado o modelo de Euler-Bernoulli e foram feitas as simulações numéricas do sistema e a análise do seu comportamento, devido à variação da frequência de excitação gerada pelo came-seguidor. Os gráficos simulados foram desenvolvidos utilizando o programa "wxMaxima" e foram utilizados para validar o sistema de simulação do aplicativo em Labview.

\section{METODOLOGIA}

\subsection{Análise do modelo da viga}

Para o modelo da viga estudada foram definidas todas as características para o sistema que será modelado com seus parâmetros. A viga tem um comprimento L, uma seção transversal constante ao longo do comprimento de dimensões b e h, sendo excitada por um sistema cameseguidor de excentricidade $e$. Essa excitação é gerada através do movimento harmônico do seguidor, $\mathrm{y}(\mathrm{t})$, definido inicialmente, tendo uma frequência $\omega_{\mathrm{f}}$. $\mathrm{O}$ movimento é transformado em força pela mola de constante elástica $\mathrm{k}_{\mathrm{m}}$, conforme esquema da Figura 2. 
CCOBENGE 2020 (C)

"Os desafios para formar hoje o engenheiro do amanhã"
$\mathrm{Ol}$ a $\mathrm{O} 3$ de dezembro

\section{Evento On-line}

Figura 2 - Viga em balanço excitada por um sistema came-seguidor

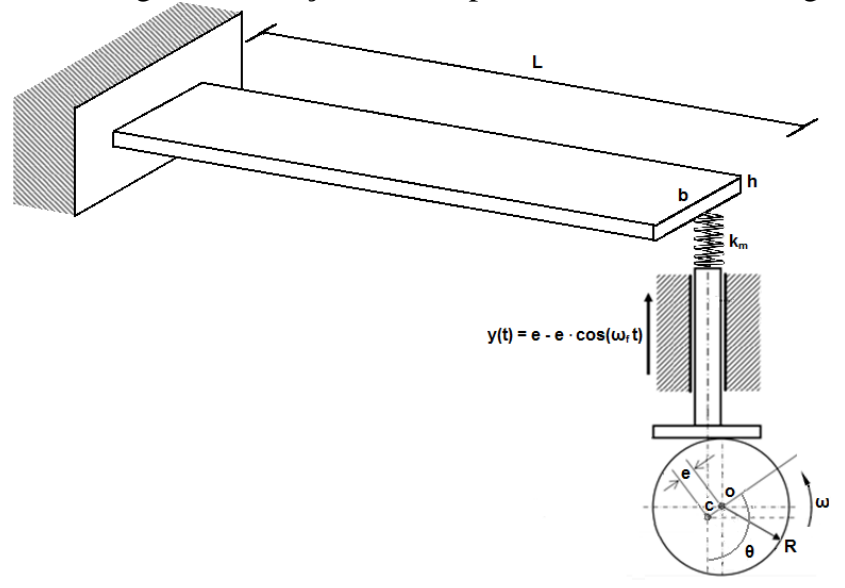

Fonte: Próprio Autor

A solução para a resposta completa da viga de Euler-Bernoulli (ALMEIDA, 2009) foi realizada aplicando os métodos da superposição, da separação de variáveis e da integral de Duhamel, resultando em:

$$
\begin{array}{r}
w(x, t)=\sum_{n=1}^{\infty} c_{1 n}\left[\cos \left(\beta_{n} x\right)-\cosh \left(\beta_{n} x\right)+\alpha_{n}\left(\operatorname{sen}\left(\beta_{n} x\right)-\operatorname{senh}\left(\beta_{n} x\right)\right)\right] \\
\left(\frac{\left(W_{n}(L) k_{m} e+C_{1 n} \delta_{n}\right)\left(1-\cos \left(\omega_{n} t\right)\right)}{\rho A b \omega_{n}{ }^{2}}-\frac{W_{n}(L) k_{m} e\left(\cos \left(\omega_{f} t\right)-\cos \left(\omega_{n} t\right)\right)}{(\rho A b)\left(\omega_{n}{ }^{2}-\omega_{f}{ }^{2}\right)}\right)
\end{array}
$$

Onde $\mathrm{C}_{1 \mathrm{n}}$ é uma constante determinada através das condições iniciais, $\rho$ é a densidade da viga, A é a área da seção transversal da viga, $\mathrm{W}_{\mathrm{n}}(\mathrm{x})$ é o n-ésimo modo de vibração, $\omega_{\mathrm{n}}$ é a nésima frequência natural da viga, $\alpha_{n}=\left[\operatorname{sen}\left(\beta_{n} L\right)-\operatorname{senh}\left(\beta_{n} L\right)\right] /\left[\cos \left(\beta_{n} L\right)+\cosh \left(\beta_{n} L\right)\right], \delta_{n}=-$ $2 \alpha_{\mathrm{n}} \mathrm{P} /\left(\beta_{\mathrm{n}} \mathrm{L}\right)$ e $\beta_{\mathrm{n}}$ são os infinitos valores encontrados através da equação de frequência, $\cos (\beta \mathrm{L}) \cosh (\beta \mathrm{L})=-1$.

Através da derivada primeira e segunda da Equação (1) em relação ao tempo, foi encontrado a velocidade e a aceleração do deslocamento da viga. Além disso, quando a frequência forçante, $\omega_{\mathrm{f}}$, se iguala a uma das frequências naturais da viga, $\omega_{\mathrm{n}}$, o sistema entra em ressonância. Para determinar a resposta desse caso, foi aplicado a regra L'Hospital (RAO, 2008) na Equação (1). Assim, a resposta do modo de vibração que está em ressonância torna-se:

$$
\begin{array}{r}
w(x, t)=\sum_{n=1}^{\infty} C_{1 n}\left[\cos \left(\beta_{n} x\right)-\cosh \left(\beta_{n} x\right)+\alpha_{n}\left(\operatorname{sen}\left(\beta_{n} x\right)-\operatorname{senh}\left(\beta_{n} x\right)\right)\right] \\
\left(\frac{\left(w_{n}(L) k_{m} e^{+}+C_{1 n} \delta_{n}\right)\left(1-\cos \left(\omega_{n} t\right)\right)}{\rho A b \omega_{n}^{2}}-\frac{W_{n}(L) k_{m} e t \operatorname{sen}\left(\omega_{n} t\right)}{\rho A b 2 \omega_{n}}\right)
\end{array}
$$

A viga simulada tem seus parâmetros mostrados na Tabela 1 e suas quatros primeiras frequências naturais apresentadas na Tabela 2 , junto com suas respectivas soluções da equação de frequência $\left(\beta_{\mathrm{n}} \mathrm{L}\right)$.

Tabela 1 - Valores para os parâmetros da viga em balanço.

\begin{tabular}{|c|c|c|c|}
\hline Parâmetro & Símbolo & Dado & Unidade \\
\hline Base & $\mathrm{b}$ & 0,0252 & {$[\mathrm{~m}]$} \\
\hline Altura & $\mathrm{h}$ & 0,0015 & {$[\mathrm{~m}]$} \\
\hline
\end{tabular}


(C) COBENCE 2020

"Os desafios para formar hoje o engenheiro do amanhã"
$\mathrm{Ol}$ a $\mathrm{O} 3$ de dezembro

\section{Evento On-line}

\begin{tabular}{|c|c|c|c|}
\hline Comprimento & L & 0,2438 & {$[\mathrm{~m}]$} \\
\hline Área & A & $3,78 \cdot 10^{-5}$ & {$\left[\mathrm{~m}^{2}\right]$} \\
\hline Momento de Inércia & I & $7,0875 \cdot 10^{-12}$ & {$\left[\mathrm{~m}^{4}\right]$} \\
\hline Módulo de Elasticidade & E & $7,1 \cdot 10^{10}$ & {$\left[\mathrm{~N} / \mathrm{m}^{2}\right]$} \\
\hline Densidade & $\rho$ & $2.800,00$ & {$\left[\mathrm{~kg} / \mathrm{m}^{3}\right]$} \\
\hline Peso & $\mathrm{P}$ & 0,2744 & {$[\mathrm{~N}]$} \\
\hline
\end{tabular}

Fonte: Próprio Autor.

Tabela 2 - Valores das quatros primeiras soluções $(\beta \mathrm{nL})$ e frequências naturais em rad/s

\begin{tabular}{|l|l|l|l|}
\hline$\beta_{1} \mathrm{~L}=1,875104$ & $\omega_{1}=128,983496$ & $\beta_{3} \mathrm{~L}=7,854757$ & $\omega_{3}=2263,335568$ \\
\hline$\beta_{2} \mathrm{~L}=4,694091$ & $\omega_{2}=808,325772$ & $\beta_{4} \mathrm{~L}=10,995540$ & $\omega_{4}=4435,234387$ \\
\hline
\end{tabular}

Fonte: Próprio Autor.

Aplicando os valores dos parâmetros da viga na Equação (1) e variando a frequência $\omega_{\mathrm{f}}$, pode-se encontrar os gráficos para os deslocamentos da viga sujeita a uma força de excitação harmônica (ANDRADE, 2009). Também foi possível identificar três casos diferentes de resposta da viga. O primeiro sendo o valor da frequência $\omega_{\mathrm{f}}$ diferente do valor de qualquer frequência natural, como mostra a Figura 3. Nela é possível observar o comportamento de um ponto qualquer da viga ao longo do tempo t, bem como o formato total da estrutura em um determinado tempo ao longo do eixo $\mathrm{x}$.

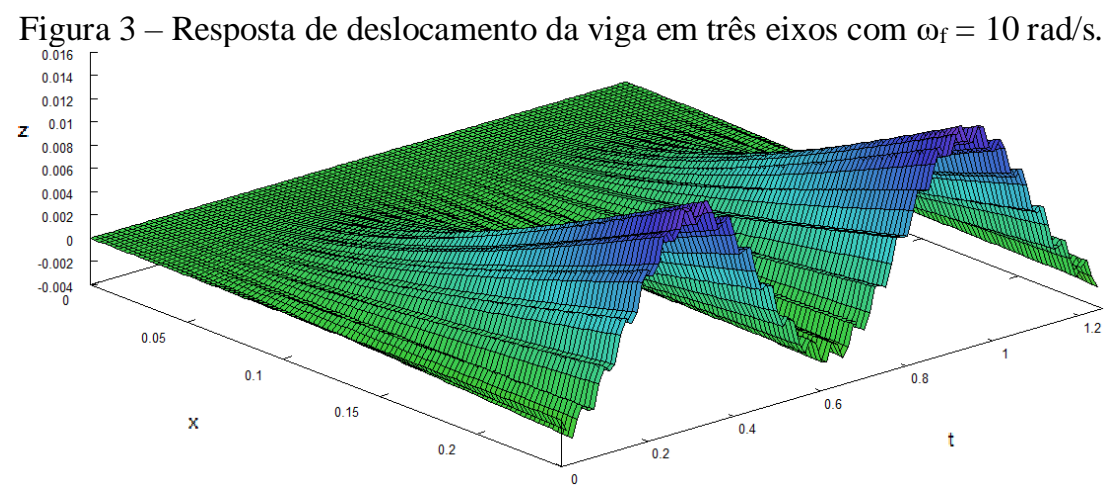

Fonte: Próprio Autor.

O segundo caso de resposta da viga acontece quando a frequência $\omega_{\mathrm{f}}$ está próxima de qualquer frequência natural, mas não igual, e é denominado de batimento (RAO, 2008). Nesse tipo de vibração a amplitude aumenta e diminui regularmente, como apresentado na Figura 4.

Figura 4 - Respostas em batimento para $\mathrm{x}=0,2338 \mathrm{~m}$ (extremidade) e $\omega_{\mathrm{f}}=798 \mathrm{rad} / \mathrm{s}$.

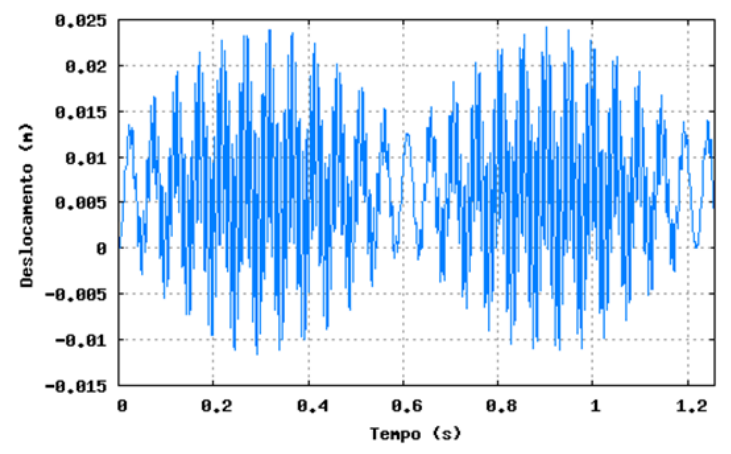

Fonte: Próprio Autor. 
O terceiro e último é o caso de ressonância onde a frequência $\omega_{\mathrm{f}}$ se iguala a qualquer frequência natural. A principal característica dessa resposta é o aumento indefinido da amplitude. Através da Equação (2) foi possível simular esse fenômeno para as duas primeiras frequências naturais, como mostrado pela Figura 5.

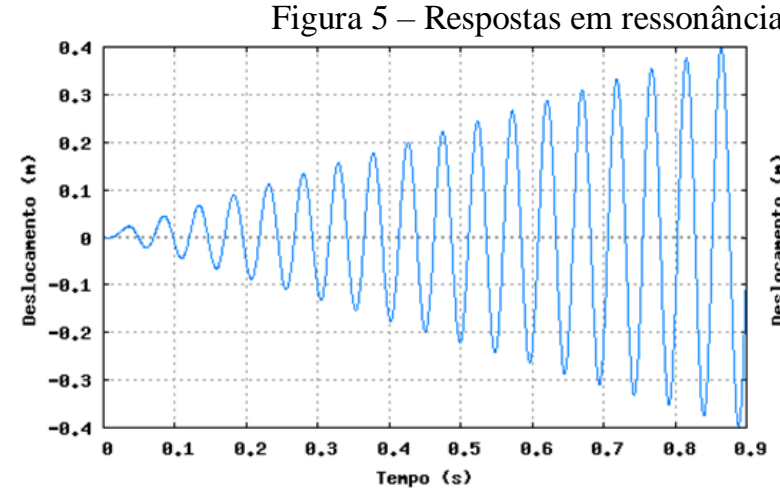

a) Resposta em ressonância para $1^{\mathrm{a}}$ frequência natural

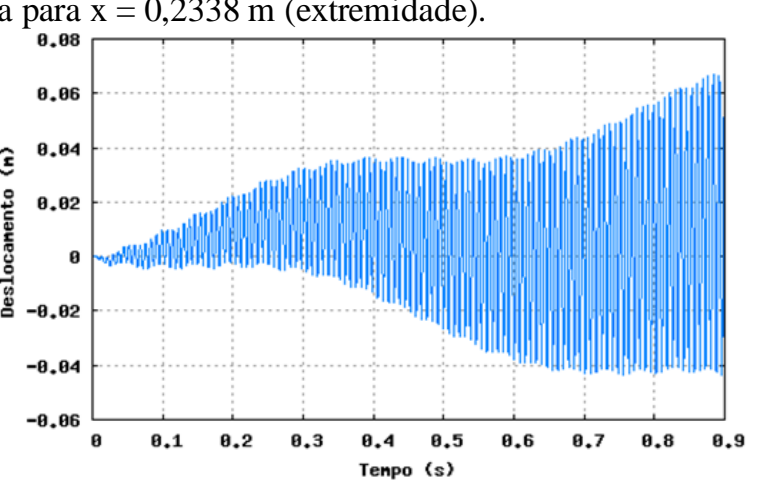

b) Resposta em ressonância para $2^{\mathrm{a}}$ frequência natural

Fonte: Próprio Autor.

As respostas para as acelerações e velocidades também foram determinadas e com isso, todos esses gráficos foram utilizados para validar as simulações realizadas no ambiente virtual LabView.

\subsection{Estruturação das rotinas no LabView}

O aplicativo desenvolvido foi nomeado como "Programa para Coleta e Visualização de Dados Experimentais" e possui quatro módulos responsáveis pela aquisição e visualização de sinais, são eles: Visualização da Aceleração, Visualização da Velocidade, Visualização do Deslocamento e Visualização da Coleta de dados. Os módulos de Visualização de Aceleração, Velocidade e Deslocamento possuem as opções de simulação e de experimento. A Figura 6, por exemplo, mostra a tela de Visualização da Aceleração para a análise experimental.

Figura 6 - Painel frontal de Visualização da Aceleração.

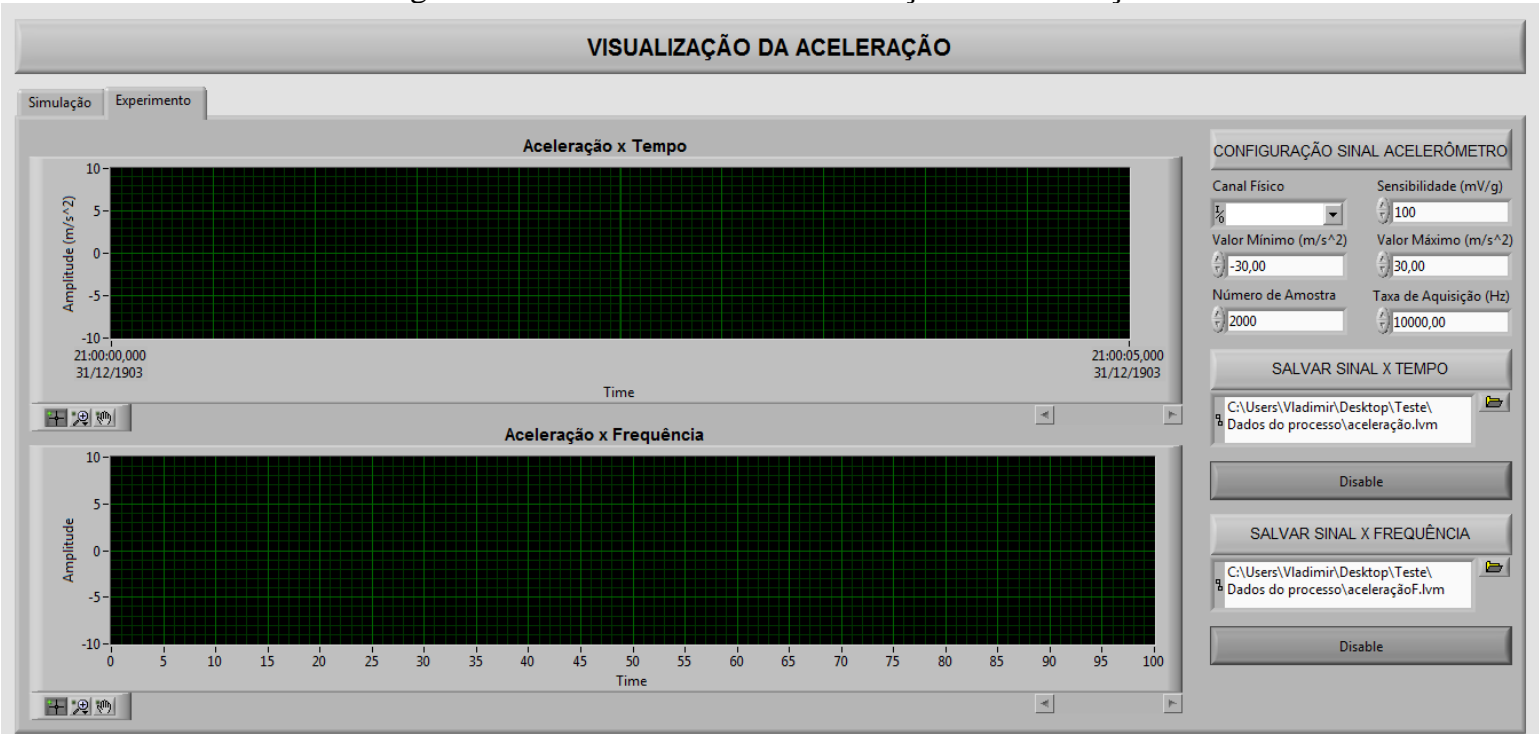

Fonte: Próprio Autor. 
CCOBENGE 2020 (C)

"Os desafios para formar hoje o engenheiro do amanhã"
$\mathrm{Ol} \mathrm{a} \mathrm{O3}$ de dezembro Evento On-line

A interface possui dois gráficos que exibem o sinal do acelerômetro, um no domínio do tempo e o outro no domínio da frequência $(\mathrm{Hz})$. Esse sinal é configurado através dos controladores numéricos situados na porção superior direita do painel. Com isso, é possível determinar o canal que fará a aquisição do sinal (BRÜEL \& KJAER, 2005), a faixa de medição desejada para a aceleração $\left(\mathrm{m} / \mathrm{s}^{2}\right)$, a sensibilidade do sensor $(\mathrm{mV} / \mathrm{g})$, o número de amostras e a taxa de aquisição $(\mathrm{Hz})$. Apenas no módulo da aceleração é permitida a realização dessa configuração, porém nos módulos de velocidade e deslocamento estarão indicados os valores determinados pelo usuário. Além do controle de sinal (MELLO, 2013), também são disponibilizados, na porção inferior esquerda da tela, o controle usado na escolha do diretório, onde serão salvos os dados do sinal no domínio do tempo e da frequência $(\mathrm{Hz})$, e os botões para habilitar o armazenamento. O diagrama de blocos desse painel está mostrado na Figura 7.

Figura 7 - Diagrama de blocos da visualização da Aceleração.

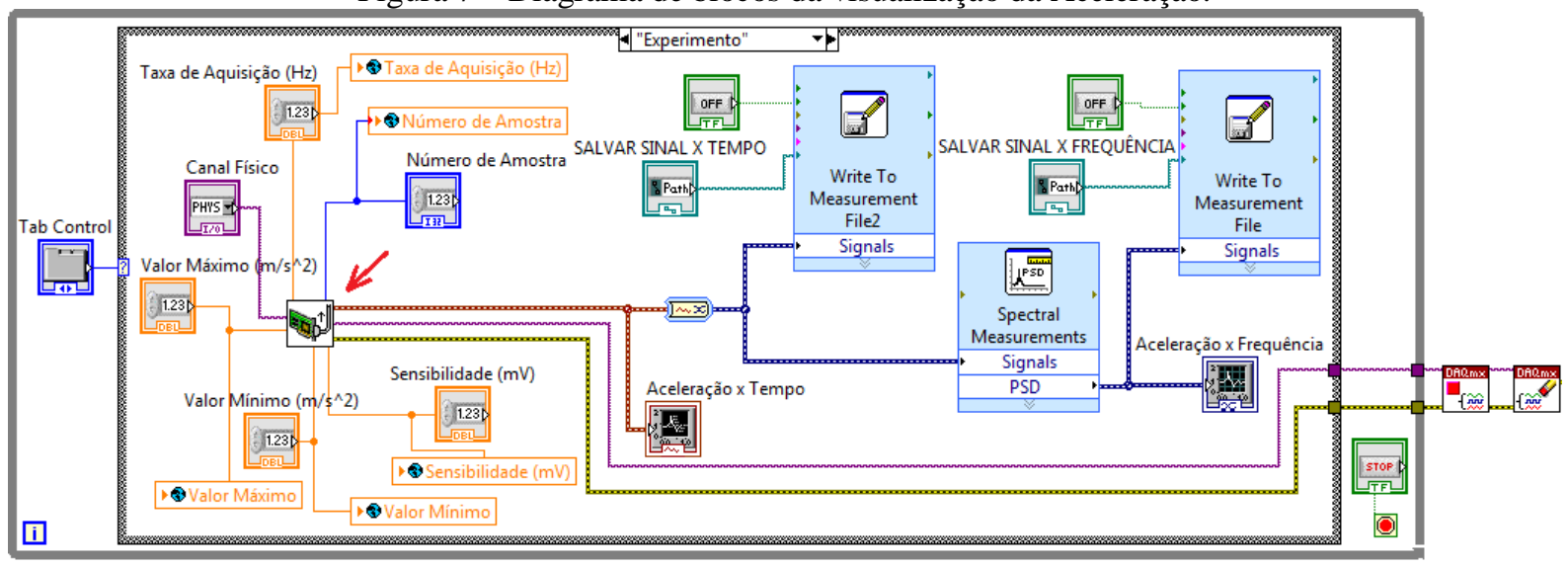

Fonte: Próprio Autor.

Diagramas de blocos semelhantes para visualização dos deslocamentos e das velocidades também foram desenvolvidos. A interface do programa principal será discutida no item a seguir.

\section{RESULTADOS E DISCUSSÕES}

O aplicativo desenvolvido "Programa para coleta e visualização de dados experimentais" possui interface principal constituída dos diversos módulos de visualização (Visualização da Aceleração, Visualização da Velocidade, Visualização do Deslocamento e Visualização da Coleta) conforme mostra a Figura (8). A janela principal do programa possui ainda um botão Stop que finaliza o programa. 
Figura 8 - Interface do Programa Principal.

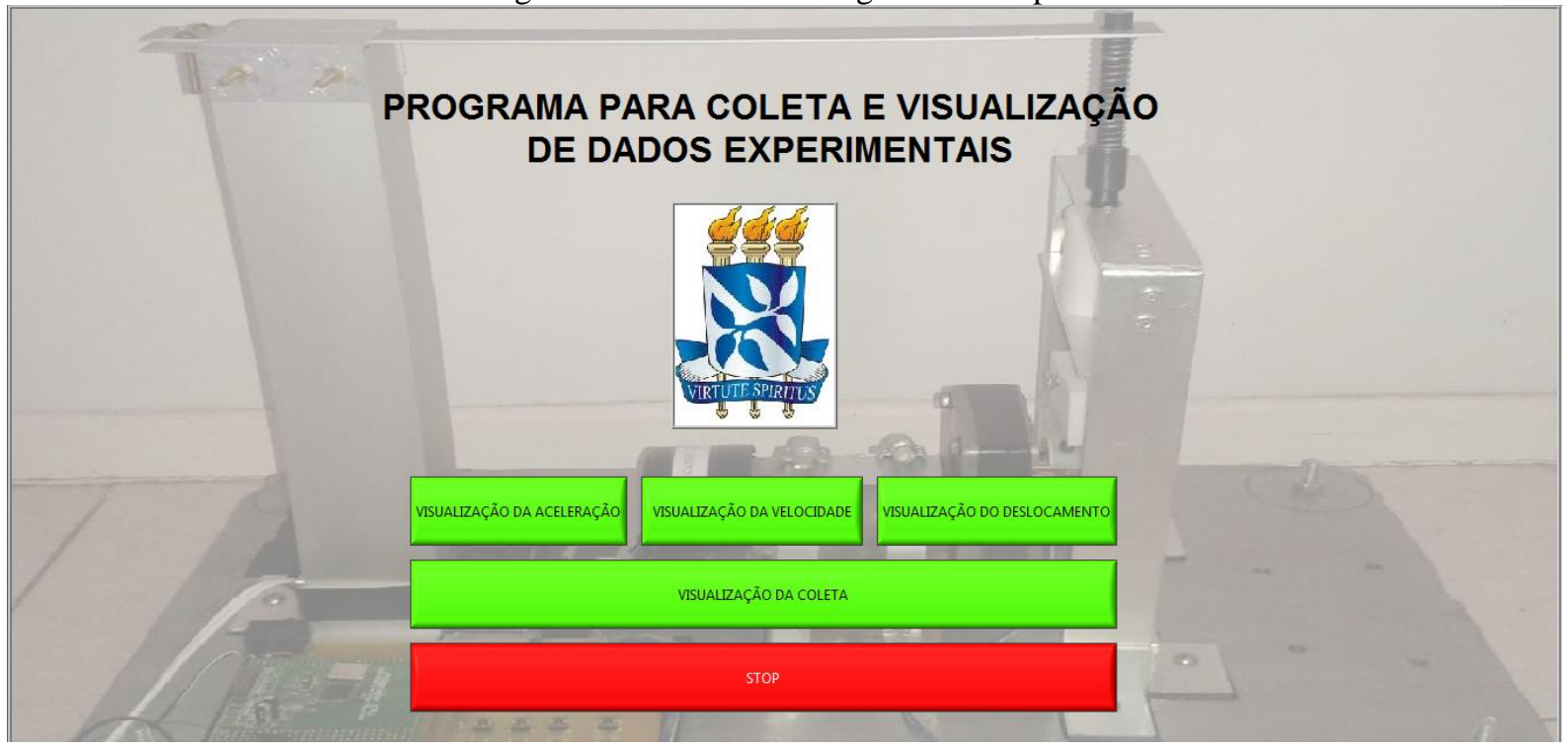

Fonte: Próprio Autor.

O diagrama de blocos do programa principal está representado na Figura 9. Neste diagrama de blocos, estão presentes os objetos responsáveis pela exibição das imagens (à esquerda) e na direita, está o loop principal que contém os subVIs referentes a cada módulo.

Figura 9 - Diagrama de blocos do programa principal

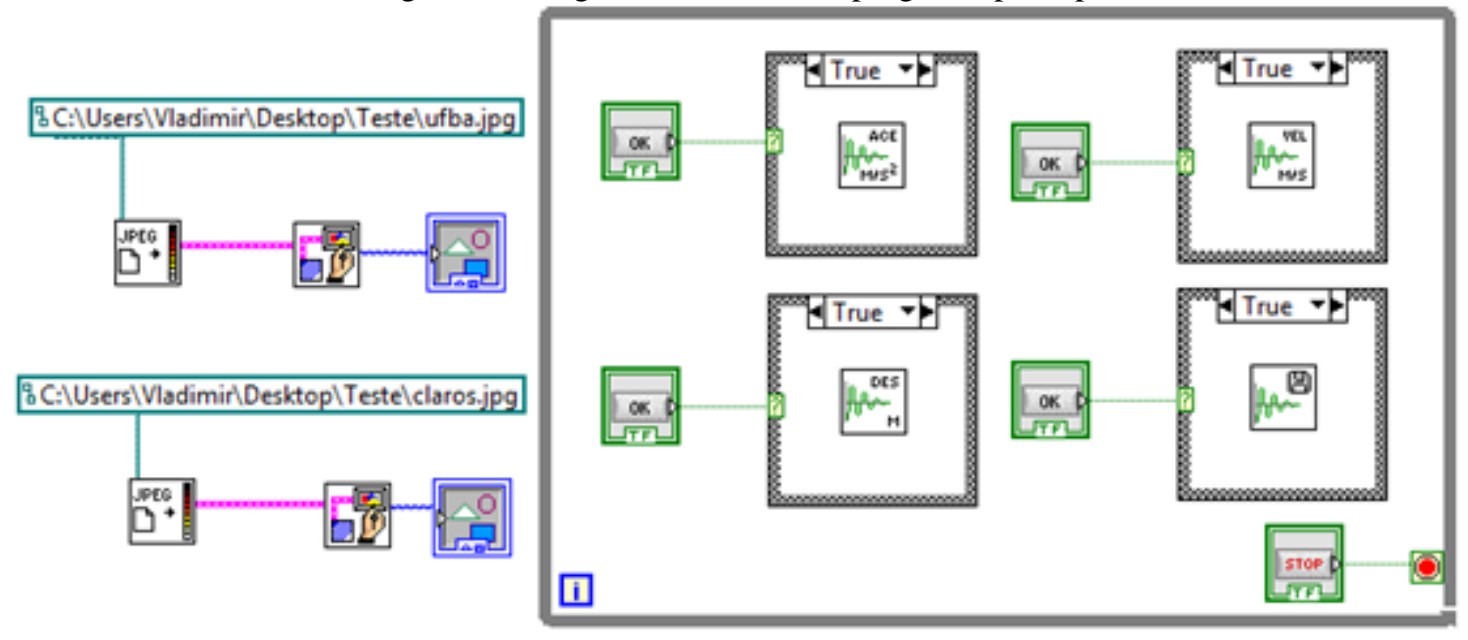

Fonte: Próprio Autor.

A Figura 10 apresenta o painel frontal do módulo de coleta. Essa interface possui dois gráficos que exibem o sinal, um no domínio do tempo e o outro no domínio da frequência $(\mathrm{Hz})$ (RAO, 2008), para a escolha pelo usuário. Na parte direita da tela são disponibilizados os controles usados na escolha do diretório, onde se encontra o arquivo salvo que se deseja exibir, tanto para o tempo quanto para frequência $(\mathrm{Hz})$. Acompanhando esses controles estão os botões para habilitar e reiniciar a exibição do sinal salvo (COELHO, 2006). 
Figura 10 - Painel frontal do módulo de Visualização da Coleta

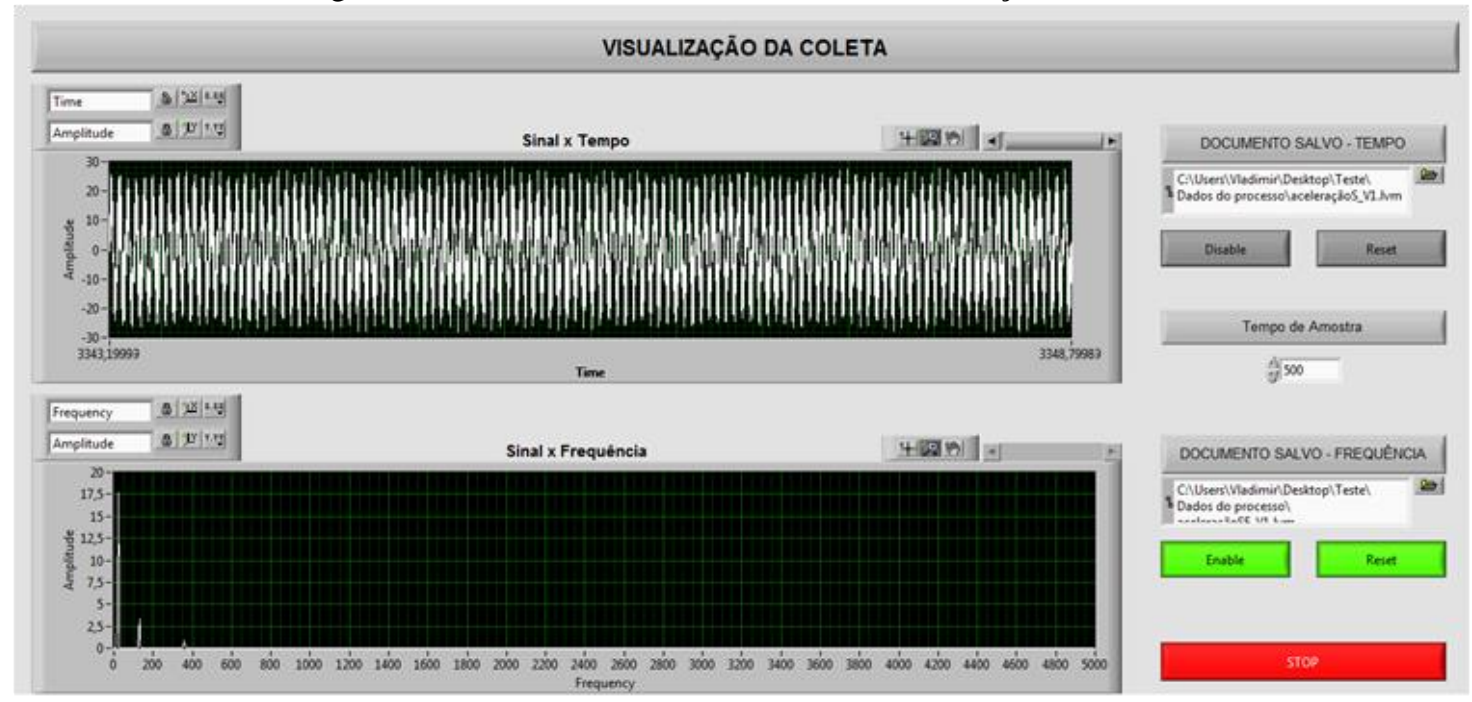

Fonte: Próprio Autor.

A opção de simulação, ilustrada pela Figura 11, mostra o comportamento de uma viga com características estabelecidas pelo usuário, além de oferecer o controle da frequência forçada e da posição de medição ao longo da viga. A resposta obtida na simulação é referente a soma dos três primeiros modos de vibração que possibilita a determinação das três primeiras frequências naturais. O usuário pode determinar os parâmetros do sistema através dos controladores numéricos localizados na porção superior direita do painel, denominado de "Características do Sistema". Esse campo permite escolher as características da viga como densidade $(\mathrm{kg} / \mathrm{m} 3)$, módulo de elasticidade $(\mathrm{N} / \mathrm{m} 2)$, altura e base da seção transversal $(\mathrm{m})$, e o peso $(\mathrm{N})$, além dos fatores que alteram a resposta apresentada no gráfico como frequência forçada ( $\mathrm{rad} / \mathrm{s})$, posição, $\mathrm{x}$, de medição $(\mathrm{m})$ e o tempo de amostragem gráfica $(\mathrm{ms})$.

Figura 11 - Painel frontal de Visualização da Aceleração para simulação.

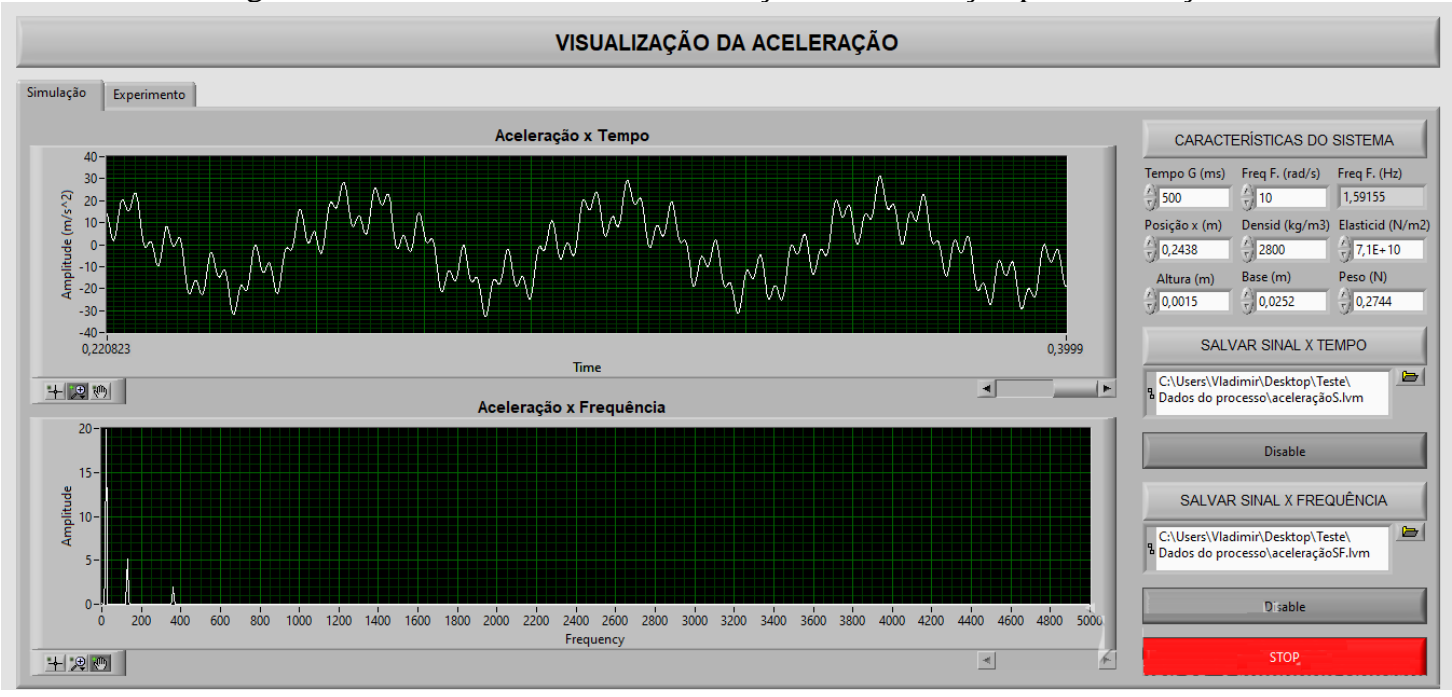

Fonte: Próprio Autor.

O diagrama da simulação é parecido com o diagrama de experimento, mostrado na Figura 7. O diferencial desse diagrama é o limitador do peso e o subVI de simulação que tem seu 


\section{COBENGE COBENGE 2020 de Educação em Engenharia}

diagrama apresentado na Figura 12. O subVI utiliza a função Simulate Signal junto com a função Formula Node que contém o código construído através da Equação (1) e Equação (2).

Figura 12 - Diagrama de bloco do subVI de simulação de sinal.

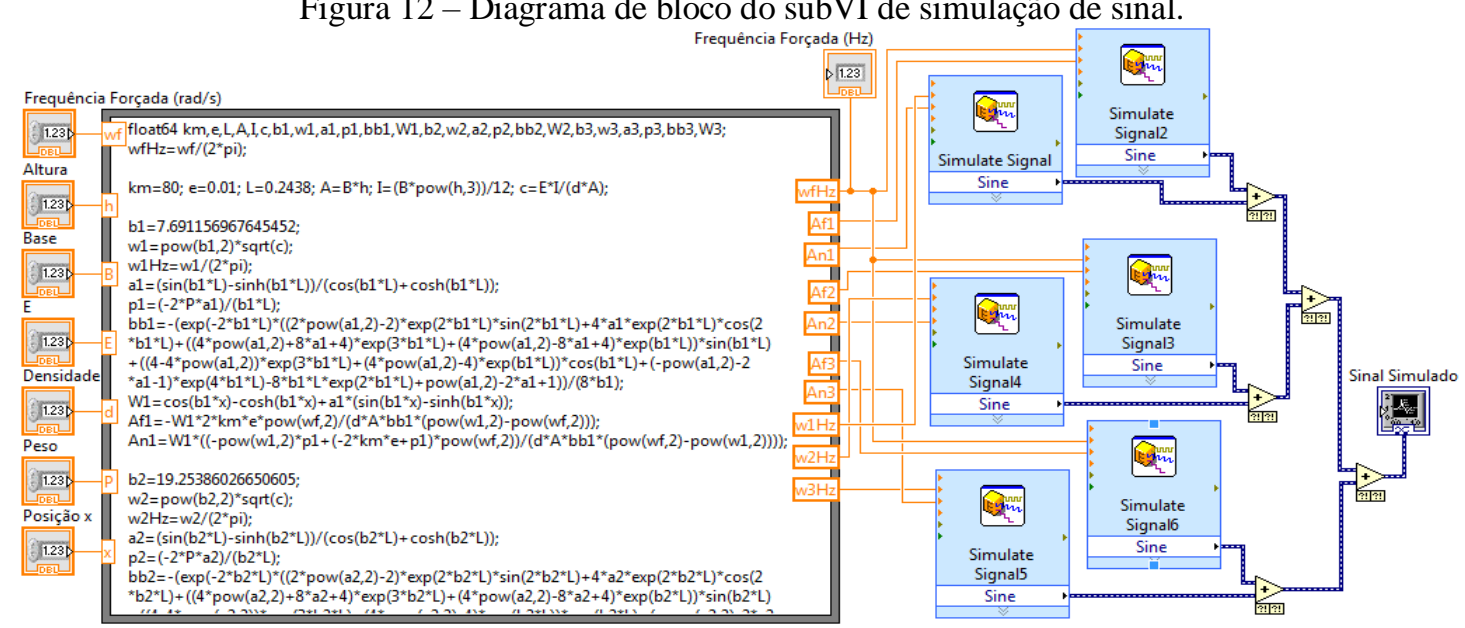

Fonte: Próprio Autor.

Na Figura 11 é mostrada também a simulação do comportamento da viga com características definidas na Tabela 1 que permitiu a comparação com os resultados obtidos no o programa "wxMaxima", pode-se notar que os espectros do gráfico de frequência estão situados exatamente nos valores encontrados das três frequências naturais.

Por fim, o programa possui uma interface gráfica que permite fácil interação como o usuário e monitoramento de dados on-line, fornecendo ainda uma série de ferramentas para medições e análises de frequências para várias aplicações. Portanto, fenômenos como o desbalanceamento e desalinhamento podem ser estudados, simulando os efeitos dos mesmos no ambiente virtual desenvolvido.

\section{CONCLUSÕES}

O aplicativo desenvolvido através do software LabView permitiu uma melhor análise dos resultados numéricos, em tempo real e de forma bem mais prática, para os três primeiros módulos de vibração. Além disso, a versão final do software se mostrou simples, autoexplicativa e capaz de guiar o usuário rapidamente através das etapas. O sistema de aquisição projetado pode ser transportado para qualquer lugar caso esse aplicativo seja instalado em um computador portátil, que possua uma placa de aquisição de sinais. Por fim, a realização do trabalho como um todo permitiu integrar os conhecimentos de várias áreas como vibrações, resistências dos materiais, projetos, eletroeletrônica e programação, e correlacionar o conhecimento teórico com o prático.

\section{REFERÊNCIAS}

ALMEIDA, Cleber Vinícius R. Equipamento para Estimação do Torque em Motores de Indução Trifásicos pelo Método do Escorregamento auxiliado pela Análise Espectral do Sinal de Corrente do Estator - Desenvolvimento e Implementação. Salvador, 2009.

Universidade Federal da Bahia. 
ANDRADE, Vinícius Santos. Análise Dinâmica de uma Viga Engastada Excitada por uma Fonte Não Ideal. São Carlos, 2009. Universidade de São Paulo.

BRÜEL \& KJAER. PRODUCT DATA. Piezoelectric Accelerometer. General Purpose DeltaTron ${ }^{\circledR}$ Accelerometers - Types 4513, 4513-001 and 4513-002. 2005. Disponível em: <http://bruel.ru/UserFiles/File/4513.pdf>. Acesso em: 16 dez. 2019.

COELHO, Raphael Rocha; ALMEIDA, Cleiton Moya de; PECCIN, Vinícius Berndsen. Controle em malha fechada da velocidade de um motor de utilizando um microcontrolador 8051. Florianópolis, 2006. Universidade Federal de Santa Catarina.

MELO, Francisco Queirós de; CARNEIRO, Joaquim. Física I. Cinemática e Dinâmica das Cames. 2008. Disponível em:

$<$ http://repositorium.sdum.uminho.pt/bitstream/1822/16931/1/Cinem\%C3\%A1tica\%20e\%20 Din\%C3\%A2mica\%20das\%20Cames.pdf>. Acesso em: 20 jan. 2020.

MELLO, Carlos Alexandre. Processamento Digital de Sinais. 2013. Centro de Informática UFPE.

NATIONAL INSTRUMENTS. LabVIEWTM - User Manual. 2003. Disponível em: <http://sukjaro.eu/ELFT-NI-palyazat/LabView_Manual.pdf>. Acesso em: 20 jan. 2020.

RAO, Singiresu.S., Vibrações Mecânicas. São Paulo, Editora Pearson Prentice Hall.2008

\title{
SOFTWARE FOR ANALYSIS OF VIBRATIONS IN A BALANCE BEAM UNDER SUBMITTED TO HARMONIC VIBRATION
}

\begin{abstract}
The behavior of rotating machines, due to its complexity, requires adequate design and analysis for the various mechanical components that make up the machine. However, determining the interaction of all subsystems of the machine, considering the large number of components and equipment, is not an easy task, since their interaction takes place through the various connection elements such as bearings and mechanical flow seals. In addition, the behavior of the main system, consisting of the rotor and support structure (bearings) can insert the effect of various phenomena such as misalignment and unbalance, in addition to the transverse, torsional and axial vibrations presented by the rotor when in operation. These phenomena can interfere with the response of the complete machine system (rotor-support structure + machine base). However, they can be investigated through mathematical models that can be adjusted based on the acquisition of experimental data obtained from the system. Therefore, this article will present the development, through the LabView software, of a graphical interface for analyzing signals from 2 test benches, which will be used as a support tool in vibration classes for the study of phenomena in rotating machines. The interface allows analyzing a system consisting of a cantilever beam excited by a cam-follower system, which will be analyzed, and a second system consisting of an axis, driven by an electric motor and supported by two bearings.
\end{abstract}

Keywords: Rotating Machines. Labview. Misalignment. Teaching Tools. 